\title{
Revealing and Sharing Malware Profile Using Malware Threat Intelligence Platform
}

\author{
Faiz Iman Djufri ${ }^{1}$ and Charles Lim ${ }^{1 *}$ \\ ${ }^{1}$ Department of Information Technology, Swiss German University, Tangerang \\ 15143 , Indonesia \\ ${ }^{*}$ Corresponding author: charles.lim@sgu.ac.id
}

\begin{abstract}
Cyber Security is an interchange between attackers and defenders, a non-static balancing force. The increasing trend of novel security threats and security incidents, which does not seem to be stopping, prompts the need to add another line of security defences. This is because the risk management and risk detection has become virtually impossible due to the limited access towards user data and the variations of modern threat taxonomies. The traditional strategy of self-discovery and signature detection which has a static nature is now obsolete in facing threats of the new generation with a dynamic nature; threats which are resilient, complex, and evasive. Therefore, this thesis discusses the use of MISP and The Triad Investigation approach to share the Indicator of Compromise on Cyber Intelligence Sharing Platform to be able to address the newt threats.
\end{abstract}

\section{Introduction}

Cyber Security is an interchange between attackers and defenders, a non-static balancing force (Schneier, 2012). As the military strategists Carl von Clausewitz, say in a wartime context, "The Defenders are in the position of the interior." You must defend yourselves against any feasible assault, even against unfulfilled attacks, while assailants must discover only one fault in penetrating the scheme (Schneier, 1998). European Union Agency For Network and Information Security reports Malware is the most common cyber threat recorded last year, involving somehow 30 percent of all occurrences of infringement in Figure 1 (ENISA, 2018).

In 2018, Identity Theft Resource Center reported Records compromised by data breach in 2018 as shown as Figure 1.2. A research by FireEye's M-Trends shows that an organisation has a average moment to find out 78 days of attack (FireEye, 2019).

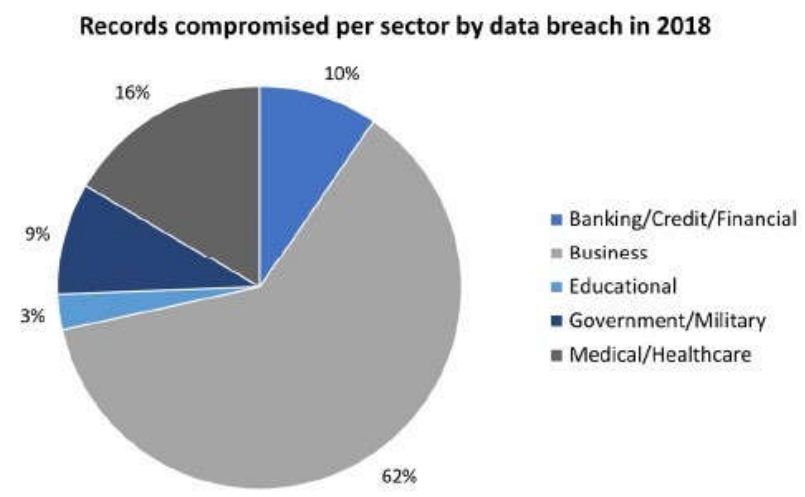

Figure 1 Records compromised by data breach in 2018, per sector (ENISA, 2018)

The increasing trend of novel security threats and security incidents, which does not seem to be stopping, prompts the need to add another line of security defenders. This is because the risk management and risk detection has become virtually impossible due to the limited access towards user data and the variations of modern threat taxonomies. The traditional strategy of self-discovery and signature detection which has a static nature is now obsolete in facing threats of the new generation with 
a dynamic nature; threats which are resilient, complex, and evasive. As a result of this new line of security defenders must include cyber attacks today. The traditional heuristic and signed static approach of safety does not match the dy namic nature of the fresh wave of risks, which are identified as evasive, durable, and complicated. Organisation, in fact, must collect and communicate cyber threat data in real time to prevent assaults or at least to implement prompt recovery from disasters. This research's contribution is to fullfil part of Detection and Analysis Process in Incident and Response procedures by analyzing the treats and sharing its information as the effort Security Analyst do, to fight with increasing of new threats and incident indicator in financial sector.

\section{Materials and Methods}

\subsection{Security Operation Center}

A Security Operation Center (SOC) is defined as a team comprised of security analysts with the objective to detect, analyze, prevent, respond to, and report on security incidents in the cyber realm (Zimmerman, 2014). The functioning of the SOC assumes that the constituency is compromised at some stage. In addition, those involved include persons who have legally access to the IT resources of the constituency. In accordance with this logic, the SOC shall be able to operate on the integrity, confidentiality and availability of constituency assets and networks without full confidence. Although the SOC must be strongly integrated with constituency IT systems, it must be isolated from compromise. There are some concern point that will become goal of operating SOC (Zimmerman, 2014):

- Achieve close to zero packet loss at specified presence surveillance points.

- Prevent the threat actor from identifying (and avoiding) the existence of (IDS and IPS) surveillance functions.

- Ensuring 100 percent of security events are delivered through SOC monitoring systems from end devices and when necessary, protect them against prying eyes. item Enhance the survivability and discourage unlawful access to SOC resources, even where constituency components are compromised.

- Protect sensitive data and records kept by SOC from disclosure

\subsection{The Incident Response Life Cycle}

As a team is composed of security analysts with the objective to detect, analyze, prevent, respond to, and report on security incidents,SOC utilize incident handling process to fulfill those objective. Well known organization like National Institute of Standards and Technology (NIST) "has responsible for developing information security standards and guidelines, including minimum requirements for Federal information system" (Cichonski et al., 2012) publish numeber, provide a publication about Incident Response Process with number document publication NIST.SP.800-61r2

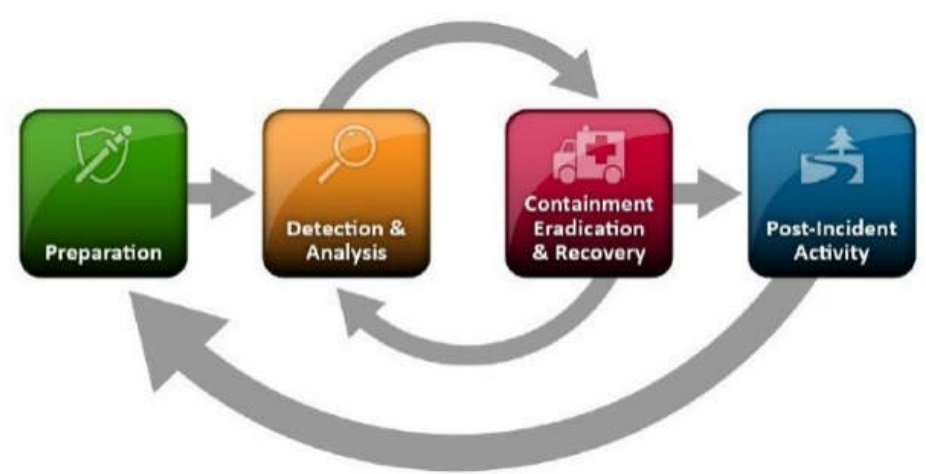

Figure 2 The Incident Response Life Cycle (Cichonski et al., 2012)

NIST Incident Handling Process, which acts as a manual for an event operator, comprises four main stages. The preparation stage is where the organization gathers the required individuals, strategies, information, and instruments to remedy an event rapidly and fully. The second stage of the method includes detecting and analyzing security events from the organization's network perimeter, target 
perimeter, system-level activity, application-level activity, or users. It also includes reporting an incident. The organization will start the stage of confinement, eradication, and restoration upon statement of an incident. The threat may proceed its path of intervention during this stage. Incident handlers often switch away and forth between the second and third phases during an incident response as new information about the threat becomes accessible. Finally, the incident response team develops the post-incident document in the post- incident operation stage and develops alternatives to enhance the method for the next incident (Cichonski et al., 2012)

\subsection{Cyber Security Threat}

In action of Incident Handling, the SOC will deal with the Cybersecurity Threat. SO (2019) define definition of Cybersecurity Threat is "an action on or through an information system that may result in an un-authorized effort to adversely impact the security, availability, confidentiality, or integrity of an information system or information that is stored on, processed by, or transiting an information system. The term does not include any action that solely involves a violation of a consumer term of service or a consumer licensing agreement". There are some common attacks on Cybersecurity Threat (Wang, 2009):

- Eavesdropping.

- Cryptanalysis.

- Password Pilfering.

- Identity Spoofing Attack.

- Buffer-Overflow Exploitations.

- Intrusion such as IP scans and port Scans.

- Denial of Service Attacks.

- Malicious Software (Malware).

\subsection{Malware}

Malware is "A program that is covertly inserted into another program or system with the intent to destroy data, run destructive or intrusive programs, or otherwise com- promise the confidentiality, integrity, or availability of the victim's data, applications, or operating system" (SO, 2019). Commonly, Malware forms include viruses, worms, Trojans, bombs, backdoors and spyware.

\subsection{Malware Analysis}

Basically, malware analysis is classified into 3 kinds:

1. Static Analysis is a malware analysis technique that looks for malicious string or signature in the body for an executable file to determine if the executable is true. Static analytical methodology basically analyzes the binary without the executable folder and is usually used for ranking by famous antivirus detection engines.

2. In general dynamic analysis is examining suspected binary by executing it. Ac- cording to Sikorski and Honig (2012), any examination performed after executing malware is called dynamic analysis. Typically dynamic analysis is performed after conducts basic static analysis and it has reached a dead end. Reason for failed static analysis such as obfuscation, packing, or the analyst have exhausted the available static analysis techniques.

3. CCode analysis is also generally classified into static analysis. Code analysis is basically a method of malware assessment that utilizes a reverse engineering technique to determine whether a specific executable file is considered malicious.

Threat Intelligence (TI), is a knowledge of threats which inform decisions for attacking prevention or reduce the gap between compromise and detection, based on evidence (MCMillan, 2013). TI commonly known as cyber threat intelligence is might kind of data that serves to illuminate the risk landscape rather than supporting particu- lar choices (Chismon and Ruks, 2015). Such as in Dalziel (2014); Steele (2014), there occur other terms. A more strict one (Dalziel, 2014) says that TI should be relevant, actionable and valuable. IT can be gathered from a number of technical sources (e.g. local sensor traffic) or from human sources (e.g. discussions in subterranean forums and peer 
communication). Thus, threat intelligence encompasses technical indicators, contexts, mechanisms, consequences and actionable advice concerning a threat existing or emerging.

Threat Intelligence is subdivided into some subdivision:

1 Strategic threat intelligence

2 Operational threat intelligence

3 Tactical threat intelligence

4 Technical threat intelligence (TTI)

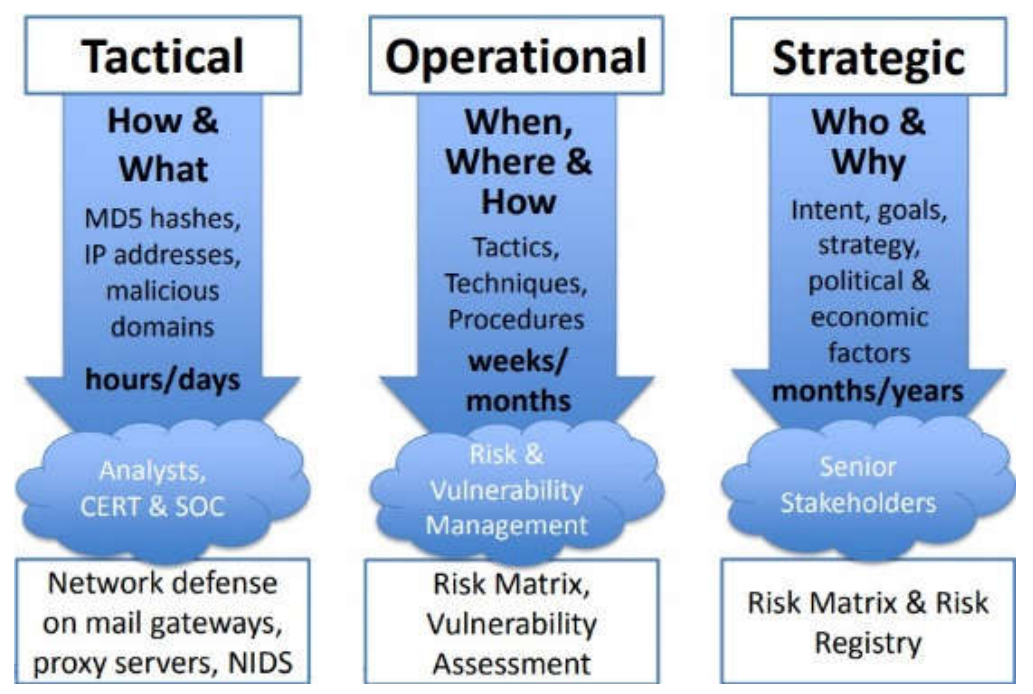

Figure 3 Type of Threat Intelligence (Irving, 2016)

\subsection{Pyramid of Pain}

The pain pyramid is an significant and elegant idea that can also be used in threat intelligence and threat hunting. The pyramid examines how hard certain attributes of their assault can alter for attackers. It also demonstrates how hard these features are for organisations to discover these attributes. Finding a file with some hash value is simple, but it is completely difficult to uncover illegitimate use in an organisation where PowerShell is frequently used. It is also trivial for assailants to build a fresh file with an other hash, but it is much difficult to relocate or alter the attacker detection method (Bianco, 2014) as shown on Figure 4.

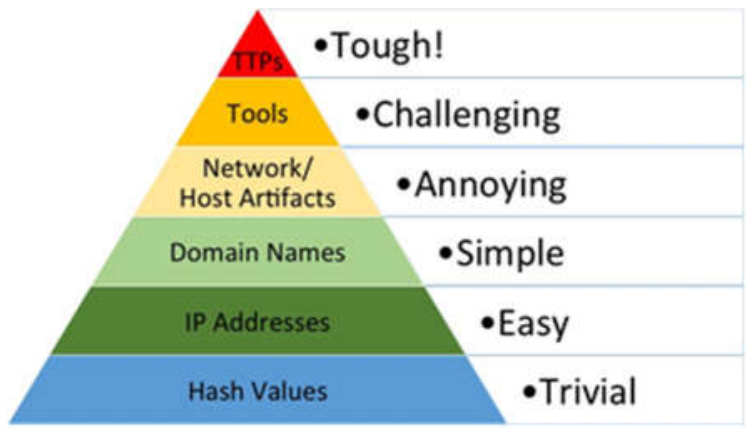

Figure 4 Pyramid of Pain

\subsection{Hunting Maturity}

The hunting maturity model (HMM) released by Bianco (2015) is one of the most quoted resources in the hunting section. This maturity model focuses on informa- tion collection, methods, the development of hypotheses, hypothesis testing instru- ments and methods, model detection and TTP automation. de Volksbank and Rabobank (2018) combined the Pyramid of Pain an Hunting Maturity Model then called it TaHiTI methodology. TaHiTI methodology provides an overview of where each stage of hunt- ing maturity operates in the pyramid and how TaHiTI should be placed in the HMM. The main focus TaHiTI 
methodology is the top 3 layers that also known as High Level IoCs. Then the other layers also known as Low Level IoCs.

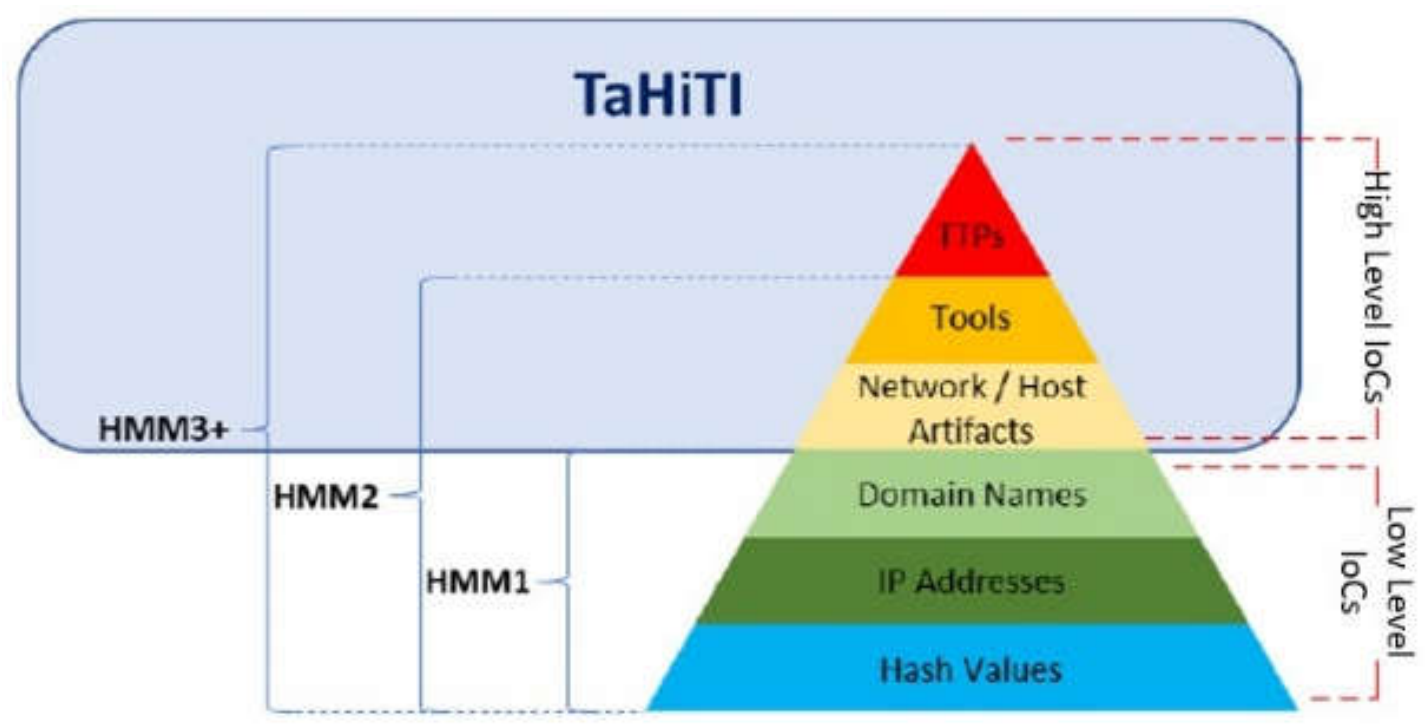

Figure 5 TaHiTI methodology

\subsection{Indicator of Compromise}

To understand better about Indicator of Compromise (IOC), one should get to know on how current Intrusion Prevention System (IPS) and Intrusion Detection System (IDS) can justify a cyber activity to be an attempt of attack launched by the adversaries. IDS and IPS share the same methods on how they can detect an activity, their detection mechanism is by parsing through previously collected data so that in the end they can generate alert based on currently flowing data compared to the collected data (Sanders To understand better about Indicator of Compromise (IOC), one should get to know on how current Intrusion Prevention System (IPS) and Intrusion Detection System (IDS) can justify a cyber activity to be an attempt of attack launched by the adversaries. IDS and IPS share the same methods on how they can detect an activity, their detection mechanism is by parsing through previously collected data so that in the end they can generate alert based on currently flowing data compared to the collected data (Sanders and Smith, 2014). Indicator of Compromise as also stated by (Pirc et al., 2016) tagged by the remnants of cyber activity that should be recognized on a network or host of an intrusion event. Indicators of Compromise leaving behind the footprints that can lead to exfiltration point of the adversaries, such as ip address, URL and file hash value.

\subsection{Malware Information Sharing Platform (MISP)}

The MISP threat sharing platform is a free and open source software that helps to share data about threat intelligence including cyber security indicators. A Threat Intelligence Platform for collecting, exchanging, distributing and correlating Targeted Attack Compromise Indicators, Threat Intelligence, Financial Fraud Information, Vulnerability Information or even Counter-Terrorism Information

\subsection{Research Framework}

This research propose Research Framework, divided into three phases of experiments:

- Data Collection, collect malware samples that related to Financial Sector.

- Analysis with Malware Analysis and use the result as input for Cyber Threat Intelligence Sharing Platform

- Creating model based information of Indicator of Compromised.

- Evaluation (Comparison) with an Cyber Threat Intelligence Sharing Platform or research in the past

- Consume and Share the result (Conclusion and Findings). 


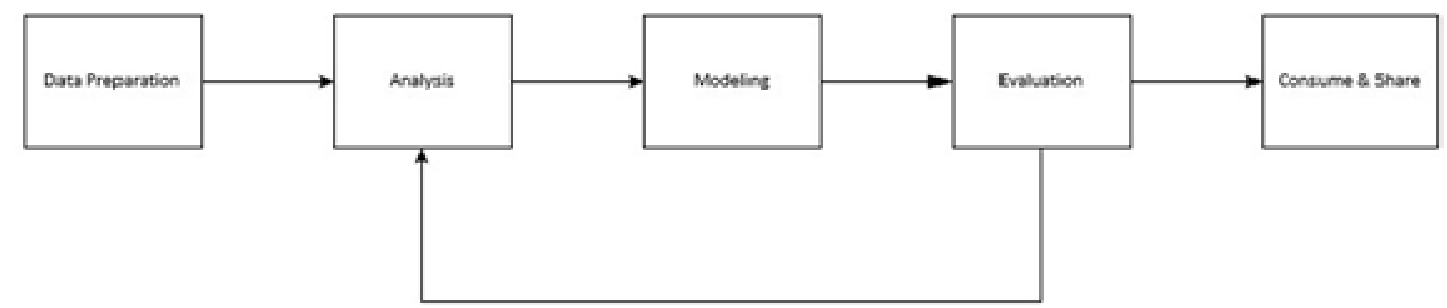

Figure 6 Research Framework

On executing their attack, Malware need some vulnerable part as target and some of them also do some evasion technique to avoid detection by Antivirus, Firewall, Sand- box etc. Based on Miller et al. (2017)'s research, there are some hardened configuration should be configured on Cuckoo Guest to make Cuckoo running well in analyzing malware activity.

(a) Adding Documents in some directory

- In "My Documents" add 5 JPGs, 1 txt, 5 PDFs, and 3 data files.

- In "My Music" add 3 MP3s.

- In "My Pictures" add 6 JPGs and 1 GIF.

- In "My Videos" add 4 MP4s.

(b) Installing New Programs

- Firefox 38.0.5.

- Notepad++ v7.

- VLC 2.2.4.

- 7-Zip 16.02.

(c) Get Recent Documents/Programs by open some documents and programs

- Open all the added documents in multiple times.

- Run each program in multiple times.

- Running some Programs.

- Open Windows explorer.

- Open Notepad.

- Disable all update services for new software.

\subsection{System Overview}

Proposed the system will use cuckoo as Malware Analysis and feed the result to Malware Information Sharing Platform (MISP)

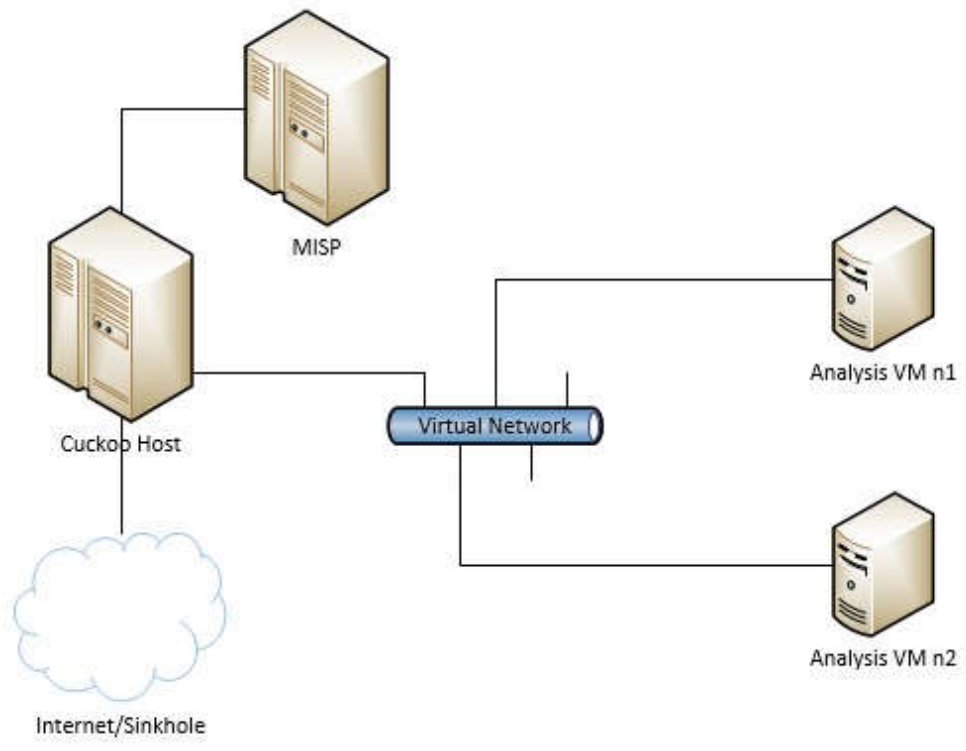




\subsection{Research Methodology Framework}

Figure 7 System Overview

\subsubsection{Data Preparation}

Dataset in this experiment utilize the last five years malware samples which attack Financial Sector during 2014 - 2019. The malware samples are collected from malware repositories such as VirusTotal, VirusShare etc. consist of 2 banking malware families such as Emotet, and Trickbot with detail:

- 30 Emotet samples,

- 29 Trickbot samples

Those Malware samples will be submitted into Cuckoo Sandbox to analyze malicious activity and get the report. By using automation API, Cuckoo feed the IoC data based on previous report into MISP. Each report of malware analysis on Cuckoo will be fed into MISP as one new event. For identifying the event, MISP give ID number on each event with others colums with details:

- Published: publishing status

- Org : organization who create event

- ID : ID number of event

- Atth : Attributes (IoC data)

- Email : username

- Date : date of inputted data

- Distribution : sharing level

As Operational Threat Intelligence, using concept the Triad Investigation is an approach to classify the data into Low Level IoC and High Level. Low Level IoC is data on intrusion response such as IP Address, Hash, Domain, URL etc. On investigation part, this work will focus on tactis, techniques, procedures of Mal- ware while executing attack (main focus of TaHITI Methodolgy). Gathering malware samples is the next step after get the list and put them into dataset which the researcher analyze use Cuckoo Sandbox as Dynamic Malware Analysis tool.

\subsubsection{Analysis}

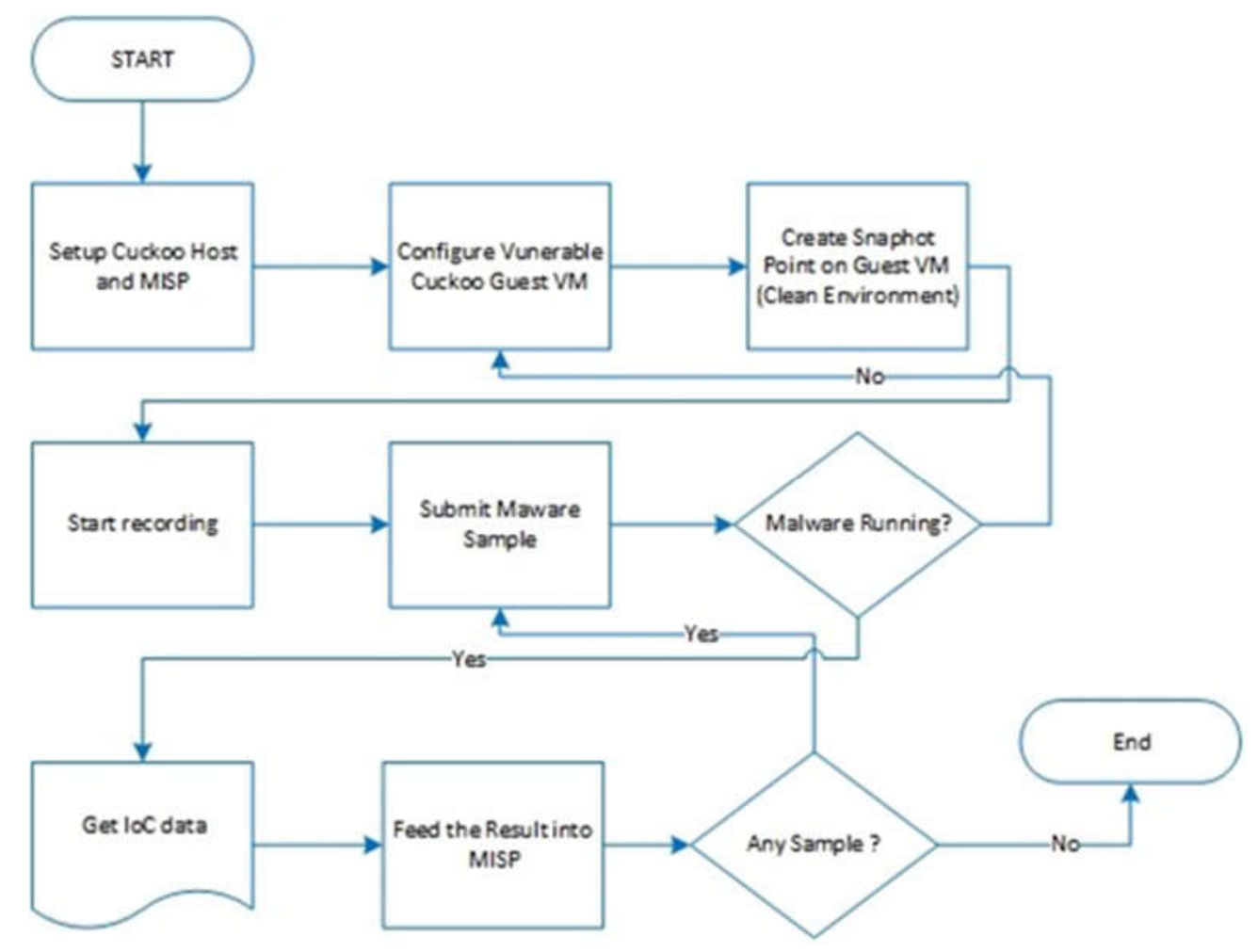

Figure 8 Phase 2- Analysis

The analysis is done following the flowchart given in Figure 8: 
- Before the malware analysis can be started, Cuckoo and MISP are setup by configurating vulnerable cuckoo using dataset collected in previous phase.

- After that snapshot on Cuckoo Guest VM is created as clean environment which will be reused to simulate the condition before malwares execute the attack.

- While malwares are submitted to Cuckoo Sandbox, the recording process of malware activity starts. When the malware activity run completely, cuckoo will generate the report and feed it to MISP.

- In the next step, with another sample the process starts again on the start recording step until all data collected in Data Preparation phase is tested.

\subsubsection{Modeling}

In this phase, scalable data model that is provided by MISP is used. It is a collection all types and features for an IoC. MISP also support import and export others data model such as STIX, OpenIoC etc for presenting IoCs informartion.

\subsubsection{Evaluation}

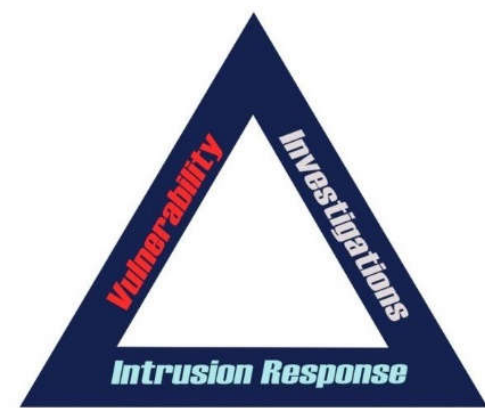

Figure 9 The Investigation Triad (Alazab et al., 2012)

This research will utilize the investigation triad for for classifying IoC data in MISP into Low Level IoC and High Level IoC.

The triad is composed of the following parts:

\section{a. Intrusion Response}

In Intrusion Response part, this research use Cuckoo Sandbox by analyzing malware samples to get data about IP Address, Port, Domain, Hash and futher called low level threat IoC. After get data, low level threat IoC information will be fed into MISP by utilizing automation API MISP for Cuckoo Sandbox. MISP will provide automatic correlating data of IoC for futhering analysis

\section{b. Investigations}

In Investigations part, this research work will do deep analysis about tactics, tech- niques and procedures (TTPs) futher called high level threat IoC. Based on result of malware analysis by using Cuckoo Sandbox, the researcher will enrich the existing data (low level threat IoCs) with high level threat IoCs information by mapping them into att\&ck mitre manually that MISP provide.

\section{c. Vulnerability}

In Vulnerability part, The vulnerabilities information will be added in CVE id for- mat on MISP. After all the data are collected consume and share it to financial sector and move another analysis with new target analysis

\subsubsection{Validation}

In this research work, the results of Malware Analysis is the most important result because it is the input data for MISP. So, the result of malware analysis has to be valid. Comparing the result on our malware analysis tool with other existing malware analysis such Cape, Hybrid-analysis, malwareanalysis etc will be used as validation method. 


\section{Results and Discussion}

We begin our method by collection respected dataset. dataset will be used as our training dataset before our framework test with malware. After collecting our training dataset, we then run the dataset in our emulation machine. Using Cuckoo Sandbox to analyze malware samples for getting data about IP Address, Port, Domain, Hash and futher called low level threat IoC. After getting the data, low level threat IoC information will be fed into MISP by utilizing automation API MISP for Cuckoo Sandbox. Hash Value of Malware. As a tool for sharing threat information, MISP can provide automatic correlation data of IoC for futher analysis. The correlation event of IoCs is shown in Table 1.

Table 1: Low Level IoC:URL

\begin{tabular}{|c|c|c|}
\hline No & URL & Correlation Found \\
\hline 1 & A & 111 \\
\hline 2 & B & 47 \\
\hline 3 & C & 47 \\
\hline 4 & D & 47 \\
\hline 5 & E & 4 \\
\hline 6 & F & 47 \\
\hline 7 & G & 17 \\
\hline 8 & H & 137 \\
\hline
\end{tabular}

Further deep analysis about tactics, techniques and procedures (TTPs) is called high level threat IoC. Based on result of malware analysis by using Cuckoo Sandbox, the researcher will enrich the existing data (low level threat IoCs) with high level threat IoCs information by mapping them manually into att\&ck mitre, that is provided by MISP.

Table 3: Low Level IoC:IP Address

\begin{tabular}{|c|c|c|}
\hline No & Destination IP Address & Correlation Found \\
\hline 1 & 118.98 .93 .11 & 20 \\
\hline 2 & 172.217 .24 .110 & 138 \\
\hline 3 & 74.125 .68 .94 & 138 \\
\hline 4 & 74.125 .24 .95 & 138 \\
\hline 5 & 74.125 .24 .94 & 138 \\
\hline 6 & 74.125 .24 .138 & 138 \\
\hline 7 & 74.125 .130 .95 & 138 \\
\hline 8 & 74.125 .130 .94 & 138 \\
\hline 9 & 65.52 .172 .55 & 63 \\
\hline 10 & 216.58 .221 .78 & 138 \\
\hline 11 & 20.189 .72 .203 & 88 \\
\hline 12 & 172.217 .27 .14 & 138 \\
\hline 13 & 172.217 .192 .94 & 138 \\
\hline 14 & 119.110 .118 .207 & 139 \\
\hline 15 & 172.217 .194 .155 & 138 \\
\hline 16 & 172.217 .194 .132 & 138 \\
\hline 17 & 172.217 .160 .42 & 18 \\
\hline 18 & 172.217 .160 .36 & 138 \\
\hline 19 & 172.217 .160 .13 & 138 \\
\hline 20 & 1157.240 .13 .6 & 138 \\
\hline & & \\
\hline
\end{tabular}




\begin{tabular}{|c|c|c|}
\hline No & Destination IP Address & Correlation Found \\
\hline 21 & 157.240 .13 .36 & 138 \\
\hline 22 & 157.240 .13 .35 & 139 \\
\hline 23 & 157.240 .13 .19 & 139 \\
\hline 24 & 13.107 .4 .50 & 76 \\
\hline
\end{tabular}

\section{Conclusion}

Based on the experimental results, several conclusions can be drawn:

1) Using Sharing Level community only on MISP is a fit Cyber Threat Intelligence Sharing model for the financial sector because they want information restricted only to financial sector companies

2) Our proposed system successfully is suitable for analyzing Emotet and Trickbot.

\section{References}

Alazab, M., Venkatraman, S., Watters, P., Alazab, M., and Alazab, A., "Cybercrime: the case of obfuscated malware," in "Global Security, Safety and Sustainability \& e- Democracy," pp. 204211, Springer, 2012.

Bianco, D., "The Pyramid of Pain," 2014, URL http://detect-respond.blogspot.com/2013/03/thepyramid-of-pain.html.

Bianco, D., “A Simple Hunting Maturity Model," 2015, URL http://detectrespond.blogspot.com/2015/10/a-simple-hunting-maturity-model.html.

Blunden, B., The Rootkit arsenal: Escape and evasion in the dark corners of the system, Jones \& Bartlett Publishers, 2012.

Cichonski, P., Millar, T., Grance, T., and Scarfone, K., "Computer Security Incident Handling Guide : Recommendations of the National Institute of Standards and Technol- ogy," Technical Report NIST SP 800-61r2, National Institute of Standards and Technol- ogy, 2012, URL https://nvlpubs.nist.gov/nistpubs/SpecialPublications/NIST.SP.800-61r2.pdf.

de Volksbank, R. v. O. and Rabobank, M. B., "DEF-TaHiTI- Threat-Hunting-Methodology.pdf," FI-ISAC NL Publication, 2018, URL https:/www.betaalvereniging.nl/wp-content/uploads/ DEF-TaHiTI-Threat-Hunting-Methodology.pdf

ENISA, "ENISA Threat Landscape Report 2018," , 2018, URL https://www.enisa. europa.eu/publications/enisa-threat-landscape-report-2018.

Fernandez, G., Nieto, A., and Lopez, J., "Modeling Malware-driven Honeypots," in Lopez, J., Fischer-Hübner, S., and Lambrinoudakis, C. (editors), "Trust, Pri- vacy and Security in Digital Business," volume 10442, pp. 130-144, Cham: Springer International Publishing, 2017, URL http://link.springer.com/10.1007/978-3-319-64483-7_9.

FireEye, "M-Trends 2019,", 2019, URL content.fireeye.com.

Irving, R., "irving-threat-intelligence-sharing.pdf,",2016, URL https://www.first.org/resources/papers /munich2016/ irving-threat-intelligence-sharing.pdf.

Kime, B., "Cyber Threat Intelligence Support to Incident Handling," p. 31, 2017. Meier, R., Scherrer, C., Gugelmann, D., Lenders, V., and Vanbever, L., "FeedRank: A tamper- resistant method for the ranking of cyber threat intelligence feeds," in "2018 10th International Conference on Cyber Conflict (CyCon)," pp. 321-344, Tallinn: IEEE, 2018, URL https://ieeexplore.ieee.org/document/8405024/.

Miller, C., Glendowne, D., Cook, H., Thomas, D., Lanclos, C., and Pape, P., "In- sights gained from constructing a large scale dynamic analysis platform," Digital In- vestigation, volume $22 \mathrm{pp}$. S48-S56, 2017, URL https://linkinghub.elsevier.com/retrieve/pii/S1742287617301949.

Moser, A., Kruegel, C., and Kirda, E., "Limits of static analysis for malware detection," in "Computer security applications conference, 2007. ACSAC 2007. Twenty-third an- nual," pp. 421-430, IEEE, 2007. 
Noor, U., Anwar, Z., Amjad, T., and Choo, K.-K. R., "A machine learning-based FinTech cyber threat attribution framework using high-level indicators of compro- mise," Future Generation Computer Systems, volume 96 pp. 227-242, 2019, URL ttps://inkinghub.elsevier.com/retrieve/pii/S0167739X18326141.

Roberto and Rodriguez, J., "How to implement and use the MITRE ATT\&CK frame- work - TOP CYBER NEWS,", 2019, URL https://www.topcybernews.com/ how-to-implement-and-usethe-mitre-attck-framework

Sikorski, M. and Honig, A., Practical malware analysis: the hands-on guide to dissect- ing malicious software, no starch press, 2012.

SO, I., "Automated Cyber Threat Intelligence Sharing," 2019, URL https://www.isao.org/storage/2019/04/ISAO-300-2-Automated-Cyber-Threat-intelligenceSharing.pdf .

Verma, A., Rao, M., Gupta, A., Jeberson, W., and Singh, V., "A literature review on malware and its analysis," International Journal of Current Research and Review, vol- ume 5(16) pp. 71-82, 2013.

Wagner, C., Dulaunoy, A., Wagener, G., and Iklody, A., "MISP: The Design and Im- plementation of a Collaborative Threat Intelligence Sharing Platform," in "Proceed- ings of the 2016 ACM on Workshop on Information Sharing and Collaborative Se- curity - WISCS'16," pp. 49-56, Vienna, Austria: ACM Press, 2016, URL http: //dl.acm.org/citation.cfm?doid=2994539.2994542.

Wang, J., Computer network security: theory and practice, Beijing: Higher Education Press, 2009, oCLC: 845386845.

Ye, Y., Li, T., Adjeroh, D., and Iyengar, S. S., "A survey on malware detection using data mining techniques," ACM Computing Surveys (CSUR), volume 50(3) p. 41, 2017.

Zimmerman, C., "Ten Strategies of a World-Class Cybersecurity Operations Center", MITRE Corporation, 2014 